Journal of

Molecular Microbiology and Biotechnology
Anaerobic hydrocarbon biodegradation 93

Archaea 74

Aromatics 181

Atrazine 212

Bacteria 74

Benzene 93

Benzylsuccinate synthase 93

Biocatalysis 139, 199

Biodegradation 74, 139, 152, 190

Biofilter 190

Bioremediation 199

- of dioxins 152

- enhancement 181

Biotransformation 152

Bph A 139

Cyanide 199
Ascenzi, P. 199

Ayala, M. 172

Barriault, D. 139

Chang, Y.-S. 152

Cipollone, R. 199

Fayolle-Guichard, F. 190

Foght, J. 93

Greer, C.W. 190

Guiot, S. 190

Hirose, S. 212

Imperi, F. 199

Kawahigashi, H. 212

Le Borgne, S. 74

Lopes Ferreira, N. 190

Lyew, D. 190

Maciel, H. 190
Mathis, H. 190

Monot, F. 190

Ohkawa, H. 212

Ohkawa, Y. 212

Pagilla, K.R. 181

Paniagua, D. 74

Pickard, M.A. 172

Pieper, D.H. 121

Seeger, M. 121

Stark, B.C. 181

Sylvestre, M. 139

Tomao, P. 199

Urgun-Demirtas, M. 181

Vazquez-Duhalt, R. 71, 74, 172

Vézina, J. 139

Visca, P. 199

\title{
Subject Index Vol. 15, No. 2-3, 2008
}

\section{CYP1A1 212}

CYP2B6 212

Environmental pollution 212

Fumarate addition 93

Halophile 74

Hypersaline 74

In situ bioremediation 93

Laccase 172

Lignin peroxidase 172

Manganese peroxidase 172

Metabolism 121

Metolachlor 212

Mycobacterium austroafricanum 190

Naphthalene 93

Natural attenuation 93

Organic pollutant 74

P450 212
PCB 139

Perlite 190

Phylogeny 139

Polychlorinated dibenzofurans (PCDFs) 152

- dibenzo- $p$-dioxins (PCDDs) 152

- biphenyls 121

Polycyclic aromatic hydrocarbons 93

Pseudomonas aeruginosa rhodanese 199

16S rRNA gene primers 190

Redox 172

Reductive dehalogenation 121

Rieske non-heme iron oxygenases 121

Stability 172

Sulfurtransferase 199

Toluene 93

Vitreoscilla hemoglobin 181 